\title{
Les rejets atmosphériques et liquides en milieu hospitalier*
}

\author{
J.C. ARTUS, P. FAUROUS**
}

(Manuscrit reçu le 11 mai 1994)

RÉSUMÉ En France, les 250 services de médecine nucléaire reçoivent, en moyenne, 6560 GBq par semaine de radionucléides en sources non scellées. De cette utilisation in vivo et in vitro résultent quelques rejets lors de la préparation des radiopharmaceutiques, des déchets radioactifs solides et, pour l'essentiel de l'activité administrée, une dispersion par le patient dans son environnement. Les rejets directs dans l'environnement, sous forme d'effluents gazeux, sont quasi inexistants et, sous forme liquide, sont très limités ; ils se pratiquent après décroissance en cuve de stockage et contrôle des dispositions réglementaires lors de leur vidange. Les contraintes liées au tri, à la gestion, à l'enlèvement des déchets radioactifs à vie longue par I'ANDRA, sont l'objet de procédures bien établies. La source de rejets la plus importante est due aux patients, elle correspond à plus de $80 \%$ de l'activité utilisée in vivo à des fins diagnostiques ou thérapeutiques. Il faut noter que les radionucléides utilisés sont, le plus souvent, de très courte période et appartiennent au groupe de faible toxicité $\left({ }^{99} \mathrm{mTc}: 6 \mathrm{~h}\right.$, 133Xe : $5,4 \mathrm{j},{ }^{201} \mathrm{Tl}: 3,4 \mathrm{j}$ ). Par contre, l'iode 131de radiotoxicité élevée (groupe 2) doit être considéré à part ; lors de son administration thérapeutique à de fortes activités, de l'ordre de $3,7 \mathrm{GBq}$, le patient est d'abord retenu en zone contrôlée, notamment pour le recueil de ses urines, mais à sa sortie il reste encore source de rejets.

ABSTRACT In France, weekly, 250 nuclear medicine departments receive an average of $6560 \mathrm{GBq}$ of unsealed sources of radionuclides. In vivo and in vitro uses result in few releases when radiopharmaceuticals are prepared, radioactive wastes and, for most administrated activities, in dispersal by the patient into his environment. There are practically no direct gaseous effuents into the environment and liquid releases are very limited and occur after decay in storage vessels and regulatory control at the time of drainage. Constraints linked to the management and collection of long-lived radionuclides by ANDRA are subject to well established procedures. Most releases originate from patients, i.e. over $80 \%$ of the activities used in vivo for diagnostic or therapeutic purposes. Most radionuclides have short half-lives and belong to the low-toxicity class ( $99 \mathrm{mTc}: 6 \mathrm{~h}$, 133Xe : $5.4 \mathrm{~d}, 201 \mathrm{Tl}: 3.4 \mathrm{~d})$. Iodine 131 widely used for therapeutic purposes has a high radiotoxicity (class 2 ) and must be considered separately ; when high activities are delivered - about $3.7 \mathrm{GBq}$ - the patient is maintained in controlled area, mainly for urine collection, but he remains a source of releases after his exit.

\footnotetext{
* Communication présentée aux journées SFRP "Seuils pratiques en radioprotection", Saclay, INSTN, 7-8 décembre 1993.

** Services de médecine nucléaire du CRLC Val D'Aurelle-Paul Lamarque, Montpellier, et du CHU, Nîmes, Parc Euromédecine, 34298 Montpellier Cedex 5.
} 


\section{Introduction}

L'utilisation courante de radionucléides en sources non scellées en milieu hospitalier est récente, elle date des années soixante et reste limitée aux services de médecine nucléaire (auparavant dénommés laboratoires de radio-isotopes). Les techniques de cette spécialité recouvrent trois types d'activité, les dosages de laboratoire utilisant le marqueur radioactif, l'administration aux patients d'un radiopharmaceutique à visée diagnostique pour exploration fonctionnelle et thérapeutique pour irradiation métabolique, dans certaines indications.

\subsection{La radioanalyse}

L'utilisation de radionucléides artificiels en sources non scellées, dans les techniques de laboratoire de radioanalyses [1], permet le diagnostic in vitro effectué sur des prélèvements biologiques. Ces techniques sont à l'origine de nombreux dosages, hormonaux, vitaminiques, enzymatiques, de médicaments, de toxiques, de marqueurs tumoraux, etc. Le radionucléide utilisé pour ces dosages est presque exclusivement aujourd'hui l'iode 125 ; d'autres traceurs, le carbone 14 , le tritium, le phospore 32 , le soufre 35 , ne sont qu'exceptionnellement employés, en tout cas en routine.

\subsection{Le diagnostic in vivo}

L'administration des radioéléments in vivo pour détecter des anomalies de fonctionnement d'organes conduit à la réalisation de scintigraphies. La médecine nucléaire se distingue des autres disciplines d'imagerie par son côté fonctionnel; il s'agit en effet de l'étude de la répartition d'un traceur, spécifique d'un organe, d'une fonction, après qu'il ait été marqué par un radioélément artificiel émetteur gamma. L'étude dans le temps et dans l'espace de la distribution du radiopharmaceutique apporte des renseignements sur la cinétique de la captation cellulaire, sur les paramètres d'une fonction, et, accessoirement, sur des considérations morphologiques d'un organe [2-4].

\subsection{L'irradiation métabolique}

A côté de ces techniques de diagnostic, la médecine nucléaire est aussi un moyen de traitement par irradiation dans quelques indications [5] : pathologies thyroïdiennes bénignes et malignes, certaines tumeurs et métastases, affections articulaires rhumatologiques... Ces traitements ont pour objet l'irradiation métabolique due à des émetteurs bêta que l'on a su amener au sein de la structure, de l'organe, de la cellule dont on souhaite la destruction. On utilise de l'iode 131 pour le traitement des états hyperfonctionnels de la thyroïde comme pour le traitement des métastases des cancers thyroïdiens, du strontium 89 pour les métastases osseuses du cancer de la prostate, de l'yttrium 90 ou de l'erbium 169 pour le traitement des tumeurs cavitaires cérébrales; les radionucléides artificiels, en sources non scellées, sont encore utilisés à des fins théra- 
peutiques dans certaines affections articulaires pour stériliser les proliférations des poches synoviales et en hématologie pour traiter certaines polyglobulies par le phosphore 32 .

\section{Organisation de la radioprotection pour la gestion des effluents en médecine nucléaire}

Les services de médecine nucléaire doivent satisfaire aux dispositions réglementaires [6] assurant une organisation correcte de la radioprotection [7] pour le personnel qui y travaille mais aussi pour l'environnement et le public.

\subsection{Les autorisations}

Les services de médecine nucléaire doivent obtenir une autorisation de création délivrée après considération des conditions sanitaires locales et des besoins spécifiques puisqu'il s'agit d'équipement en matériel lourd pour lequel une autorisation distincte doit être obtenue. Une fois cette autorisation de principe délivrée par le ministère de la santé, il convient d'assurer la conformité des installations pour le respect de la réglementation; enfin, l'activité de ces services est liée à l'autorisation personnelle, renouvelable, accordée à la personne apte à commander, détenir, utiliser des radioéléments artificiels en sources non scellées. Cette autorisation est délivrée par la direction générale de la santé après instruction du dossier par l'Office de protection contre les rayonnements ionisants, l'OPRI, et par la Commission interministérielle des radioéléments artificiels, la CIREA.

Les services de médecine nucléaire n'ont pas tous les mêmes autorisations ; ils sont distingués en différentes classes en fonction de l'activité maximale qu'ils peuvent utiliser :

$-1^{\text {re }}$ Classe : activité supérieure à $11,1 \mathrm{GBq}(300 \mathrm{mCi})$;

$-2^{\mathrm{e}}$ Classe : activité comprise entre 1,1 et $11,1 \mathrm{GBq}(30$ à $300 \mathrm{mCi})$;

$-3^{\mathrm{e}}$ Classe : activité inférieure à $1,1 \mathrm{GBq}(30 \mathrm{mCi})$;

$-4^{\mathrm{e}}$ Classe : activité inférieure à $37 \mathrm{MBq}(1 \mathrm{mCi})$.

Le nombre de services de médecine nucléaire reste limité ; jusqu'en 1985 l'utilisation des radionucléides artificiels en sources non scellées restait limitée au secteur hospitalier public; depuis 1986, des services privés ont pu être ouverts ; malgré cet élargissement au secteur libéral, le territoire reste mal couvert et la France est relativement sous-équipée par rapport aux pays européens voisins. La répartition des services de médecine nuclaire en fonction du secteur d'activité et des établissements où elle est pratiquée traduit le nombre restreint de ces services (Tab. I). 
TABLEAU I

Répartition des services en fonction de leur activité et de leur structure d'implantation (décembre 1993)

Distribution of departments as a function of their activities and location structures

\begin{tabular}{|c|c|c|c|c|c|}
\hline $\begin{array}{l}\text { In vitro } \\
\text { seul }\end{array}$ & 43 & 18 & 3 & 11 & 75 \\
\hline $\begin{array}{l}\text { In vivo } \\
\text { seul }\end{array}$ & 16 & 27 & 3 & 39 & 85 \\
\hline $\begin{array}{l}\text { In vivo } \\
\text { In vitro }\end{array}$ & 43 & 26 & 17 & 1 & 87 \\
\hline Total & 102 & 71 & 23 & 51 & 247 \\
\hline
\end{tabular}

CHU : centre hospitalier universitaire

CHG : centre hospitalier

CAC : centre anticancéreux

\subsection{Exigences pour les effluents gazeux}

Les exigences requises pour les effluents gazeux sont rapportées dans les textes d'application de la réglementation (arrêté du 30 octobre 1981, article 8); ces effluents sont contrôlés et leur activité ne doit pas dépasser $4 \mathrm{~Bq} / \mathrm{m}^{3}$ lors de leur évacuation par des cheminées amenant assez haut en toiture ces éléments pour éviter leur recyclage. Pour gérer ces effluents gazeux potentiels, l'atmosphère de la zone contrôlée du service doit être ventilée en dépression par rapport au reste de l'établissement; à l'intérieur de cette zone, le laboratoire chaud dans lequel sont manipulées les sources doit être lui-même en dépression par rapport au reste du service et sa ventilation doit assurer 10 renouvellements par heure ; les boîtes à gants plombées doivent être en dépression par rapport à la pièce et leur atmosphère renouvelée 30 à 40 fois par heure ; l'extraction se fait à travers des filtres à charbon actifs contrôlés. Dans le cas de l'utilisation de xénon 133 pour l'exploration ventilatoire pulmonaire, une extraction spécifique est prévue dans la pièce pour recueillir au mieux l'air exhalé par le patient et son rejet est direct à la cheminée.

Les rejets de radionucléides artificiels en sources non scellées, sous forme gazeuse, sont assez rares en définitive et lorsque le xénon n'est pas utilisé, ce qui n'est pas rare, les vapeurs d'iode représentent la quasi-totalité des rejets atmosphériques.

\subsection{Exigences pour les effluents liquides}

Les dispositions requises pour les effluents liquides nécessitent, notamment dans la pièce de préparation des radiopharmaceutiques, la présence d'éviers 
chauds reliés à des cuves de stockage pour une gestion en décroissance. Selon l'arrêté du 30 octobre 1981, article 8, un contrôle à moins de $7 \mathrm{~Bq} / \mathrm{l}^{-1}$ doit être effectué pour que les cuves puissent être vidées dans le réseau dont le débit doit être, au moins, de $5 \mathrm{~m}^{3} / \mathrm{s}$; cette limite peut paraître peu adaptée car d'une part il n'est pas aisé de disposer, dans les services de médecine nucléaire, de moyens de comptage d'aussi faibles activités, d'autre part, puisque les radioéléments à vie "relativement longue" retrouvés exclusivement dans les cuves sont bien connus (iodes 125 ou 131), il paraît raisonnable de considérer comme limites d'activité volumique celles correspondant aux limites annuelles d'incorporation (LAI) respectives. Les toilettes du service, utilisées par les patients ayant reçu le marqueur et à qui il est demandé de vider la vessie avant l'examen, sont reliées au réseau à travers une fosse septique pour assurer un minimum de dilution et de stockage transitoire.

Les effluents liquides des sanitaires des chambres protégées où séjournent les patients ayant reçu des activités élevées d'iode 131 sont eux aussi conduits à des cuves de stockage qui reçoivent exclusivement ce radionucléide. Ces cuves sont évacuées dans les mêmes conditions.

Une aire de stockage des déchets solides mais contenant des radionucléides en phase liquide est prévue pour une gestion en décroissance des déchets de "courtes périodes" avant qu'ils ne rejoignent le circuit habituel des déchets hospitaliers alors que les déchets de longue période (supérieure à 71 jours), après entreposage, sont enlevés par l'ANDRA.

\section{Quantités annuelles de radionucléides artificiels utilisés en médecine nucléaire}

L'importance du rejet d'effluents gazeux ou liquides dépend certainement des précautions et des dispositifs nécessaires à leur gestion mais aussi et surtout de l'importance des activités consommées par les services de médecine nucléaire. En fonction de leur devenir ou de leurs caractéristiques les radionucléides employés pour les techniques de laboratoire sont distingués de ceux utilisés in vivo soit pour le diagnostic, soit pour leurs prescriptions thérapeutiques.

\subsection{Radionucléides utilisés pour les activités de laboratoire (Tab. II)}

La quasi totalité des laboratoires utilisent seulement de l'iode 125 et quelques unités d'autres marqueurs en plus. Pour ces autres radioéléments, le carbone 14 et le tritium ont des périodes qui ne permettent pas leur élimination par la gestion sur place; ils doivent être pris en charge par l'ANDRA.

L'importance, relative, de l'iode 125 est due à la grande prépondérance des dosages de routine utilisant presque exclusivement ce marqueur. Les autres radioéléments sont surtout nécessaires aux travaux de recherche associés à l'activité de routine des laboratoires de radioanalyses. 
TABLEAU II

Caractéristiques et activités des marqueurs utilisés, en France, par les laboratoires d'analyses ou de recherche en 1992 (source CIREA)

Characteristics and activities of the radionuclides used by analysis or research laboratories, France, 1992

\begin{tabular}{|c|c|c|}
\hline Radionue & Perrod & te \\
\hline $125 I$ & $59,9 \mathrm{j}$ & 134 \\
\hline${ }^{14} \mathrm{C}$ & $5730 a$ & 13 \\
\hline${ }^{51} \mathrm{Cr}$ & $27,7 \mathbf{j}$ & 9 \\
\hline${ }^{3} \mathrm{H}$ & $12,3 \mathbf{j}$ & 8 \\
\hline${ }^{32} \mathrm{P}$ & $14,3 \mathrm{j}$ & 3 \\
\hline${ }^{35} \mathrm{~S}$ & $87,4 \mathrm{j}$ & 1 \\
\hline
\end{tabular}

\subsection{Radioéléments utilisés pour l'administration aux patients (Tab. III)}

Les activités administrées sont notablement plus importantes que celles utilisées en techniques de laboratoire.

L'activité du technétium $99^{\mathrm{m}}$ utilisée en médecine nucléaire représente, à elle seule, plus de $85 \%$ de l'activité totale. Ce radioélément artificiel est, de loin, le plus utilisé et la médecine nucléaire lui doit son essor car il présente de multiples avantages :

- il est produit sur place dans les services par élution d'une colonne de molybdène 99 ,

- ses propriétés chimiques en font un marqueur polyvalent,

- c'est un émetteur gamma pur dont l'énergie est adaptée aux détecteurs à scintillations,

- sa faible période permet l'utilisation de fortes activités, de l'ordre de $740 \mathrm{MBq}(20 \mathrm{mCi})$, sans entraîner une exposition trop importante du patient,

- sa gestion en décroissance est rapide et ne pose pas de problème : en moins de 3 jours, il reste moins du 1/1000 de l'activité considérée.

Le technétium $99 \mathrm{~m}$ avec le xénon 133, l'iode 131 et le thallium 201, représentent plus de $99 \%$ de l'activité totale. Parmi ces quatre radioéléments les plus importants, l'iode 131, bien qu'il ne soit pratiquement plus utilisé en diagnostic mais surtout à des fins thérapeutiques, doit être considéré à part car sa radiotoxicité est élevée (groupe 2). L'importance de son activité est notable : $5,5 \%$ de l'activité totale à lui seul. En dehors de l'iode, et en raison de leur faible période, les autres radioéléments artificiels utilisés en médecine nucléaire appartiennent au groupe de toxicité faible (groupe 4) et pour quelques uns à celui de toxicité modérée (groupe 3 ). 
TABLEAU III

Caractéristiques et activités des marqueurs utilisés, en France, par les services de médecine nucléaire à des fins de diagnostic in vivo ou de traitement en 1992 (source CIREA)

Characteristics and activities used by nuclear medicine departments for in vivo diagnostic or therapeutic purposes, France, 1992

\begin{tabular}{|c|c|c|}
\hline \multicolumn{3}{|c|}{ Radionucléte $\quad$ Periode Activite (GBq) } \\
\hline 99m Tc & $6,00 \mathrm{~h}$ & 290094 \\
\hline${ }^{133} \mathrm{Xe}$ & $5,2 \mathrm{j}$ & 19500 \\
\hline 131I & $8,02 \mathrm{j}$ & 19240 \\
\hline $201 \mathrm{Tl}$ & $3.04 \mathrm{i}$ & 9926 \\
\hline${ }^{123} I$ & $13,2 \mathrm{~h}$ & 583 \\
\hline 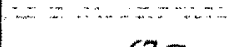 & $=-x=$ & $=-2=0$ \\
\hline${ }^{67} \mathrm{Ga}$ & $3,3 \mathrm{j}$ & 497 \\
\hline $90 Y$ & $26 i$ & 283 \\
\hline & $2,0 J$ & \\
\hline${ }^{111}$ In & $2,8 \mathrm{j}$ & 228 \\
\hline 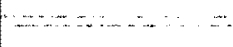 & $\therefore x=0$ & 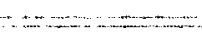 \\
\hline${ }^{186} \mathrm{Re}$ & $90,6 \mathrm{~h}$ & 220 \\
\hline $32 \mathrm{P}$ & $14,3 \mathrm{j}$ & 171 \\
\hline & $=0$. & 109 \\
\hline${ }^{109} \mathrm{Er}$ & $9,4 \mathrm{j}$ & 104 \\
\hline${ }^{51} \mathrm{Cr}$ & $27,7 \mathrm{j}$ & 65 \\
\hline
\end{tabular}

\section{Devenir des radionucléides artificiels utilisés dans les laboratoires}

Une partie du marqueur est piégée dans le complexe antigène-anticorps de la réaction immunologique, l'autre partie est libre. Globalement, $50 \%$ de l'activité environ demeure dans le tube au moment de la mesure et le reste a été évacué du tube par aspiration du surnageant lors des manipulations.

Le plus souvent, le marqueur est l'iode 125 ; en raison de sa période, le surnageant est stocké dans une cuve via les éviers chauds. Habituellement; deux cuves se remplissent en alternance sur une durée de plus d'un an. La cuve gérée en décroissance pendant le temps de remplissage de l'autre est ensuite évacuée, après contrôle de l'activité des effluents, vers le collecteur central de l'établissement. Les tubes d'analyses une fois comptés constituent ainsi une grande part des déchets radioactifs solides (non putrescibles SP ou SL selon la classification de l'ANDRA) dont la gestion en décroissance, pour l'iode 125, doit pouvoir être assurée sur place par stockage pendant deux ans environ. Ces 
déchets ne sont pas source notable $d$ 'effluents radioactifs" car ils n'ont que très peu d'activité (de l'ordre de quelques $\mathrm{kBq}$ par tube), leur gestion en décroissance est facile ; à l'issue de leur stockage, après contrôle, ils sont considérés comme déchets biologiques et finalement incinérés.

Pour les autres marqueurs, le carbone 14, le tritium, ou autre soufre 35 , les surnageant ou les mélanges organiques contenant l'activité sont entreposés dans des bonbonnes et constituent les déchets liquides (aqueux LA ou solvants LS) ; ils sont évacués, avec les tubes ou flacons constituant les déchets "solidesliquides" (SL) par l'ANDRA.

La gestion en décroissance des déchets solides putrescibles, pièces opératoires, cadavres d'animaux (SO selon l'ANDRA) se fait par leur entreposage ou stockage en état de congélation.

$\mathrm{Au}$ total la radioactivité manipulée (quelques dizaines de $\mathrm{MBq} / \mathrm{mois}$ pour l'iode 125) dans les laboratoires de radioanalyses des services de médecine nucléaire est totalement contenue dans les cuves et la production des déchets radioactifs ; leur gestion correcte permet une non dissémination; les contrôles des filtres des ventilations et des effluents des cuves avant leur vidange, garantissent la prise en compte de rejets accidentels dans l'environnement. Le passage des techniques radioisotopiques d'analyses biologiques aux méthodes non radioactives diminuerait les contraintes de gestion en décroissance mais ces techniques "froides" sont, au mieux, de qualité équivalente mais toujours beaucoup plus onéreuses.

\section{Devenir des radionucléides artificiels utilisés in vivo à des fins diagnostiques ou thérapeutiques}

A cause de la faible valeur de leur période et du délai entre la réception et l'utilisation des radioéléments, on peut évaluer à $10 \%$ environ l'ordre de grandeur de l'activité globale perdue par décroissance suite au "délai d'utilisation".

Les différentes manipulations nécessaires à la préparation des radiopharmaceutiques, les reliquats d'activité contenus dans les aiguilles, les seringues, les flacons et autres tuyauteries d'injection, sont responsables de la production des déchets radioactifs dont l'activité, au plus, correspond à $10 \%$ environ de l'activité globale commandée. Même si les activités de ces déchets sont notables, la faible et parfois très faible période physique des radionucléides permet leur gestion facile sur l'aire de stockage. Un tri sélectif notamment par la mise à part des déchets technétiés, permet d'en éliminer la plus grande partie après un temps de stockage limité (une semaine entraîne un facteur de décroissance de $10^{8}$ ). Pour le reste des déchets, une période de 8 jours, celle de l'iode 131 (la plus longue pour la grande majorité des autres radioéléments artificiels utilisés) sert de référence pour leur gestion [8]. 


\subsection{Pour la grande majorité des marqueurs}

En moyenne, $80 \%$ au moins de l'activité totale, enregistrée par la CIREA, est administrée aux patients et échappe à la zone contrôlée lorsque le patient quitte le service ; cette source potentielle d'effluents radioactifs vers l'environnement est caractérisée par :

- son importance, plus des trois quart de l'activité totale,

- un contrôle difficile assuré par les seuls conseils de radioprotection formulés aux patients.

En fonction de sa cinétique et de son absence d'affinité, il faut distinguer le cas du xénon 133 qui, administré le plus souvent sous forme gazeuse par inhalation, ne séjourne que très peu de temps dans l'organisme, sa période biologique est très faible. Il est rapidement et en quasi-totalité rejeté par expiration dans un circuit spécial évacuant de l'atmosphère en zone contrôlée du service l'essentiel de l'activité ; cette extraction spécifique est directement reliée à la cheminée du service.

En dehors de cette utilisation et pour la plupart des autres marqueurs de radiopharmaceutiques, l'élimination dans l'environnement se fait principalement par les urines avec une cinétique qui dépend de la demi-vie biologique, elle-même fonction de la pharmacocinétique du traceur. En moyenne, les effluents, essentiellement liquides, évacués par les urines, représentent de 40 à $80 \%$ de l'activité administrée. Une partie assez importante de cette activité urinaire est évacuée, avant l'examen scintigraphique, dans les toilettes du service reliées au collecteur de l'établissement (sans autre dispositif qu'une fosse septique qui sert de cuve tampon, de décroissance et de dilution) ; par la suite, les urines émises plus tardivement vont dans les toilettes des particuliers.

En définitive, la miction est la principale -source d'effluents radioactifs liquides en milieu hospitalier et c'est d'elle que peuvent provenir, hors de la zone contrôlée du service, les contaminations de l'entourage du patient.

\subsection{Cas particulier de l'iode 131}

Cette source urinaire d'effluents liquides doit être prise particulièrement en considération dans le cas de l'administration de fortes activités (3,7 GBq et plus) d'iode 131 à des fins thérapeutiques. Le cumul des urines évacuées sur $72 \mathrm{~h}$ correspond en moyenne à $80 \%$ environ de l'activité administrée et justifie l'hospitalisation et le confinement du patient en chambre protégée pendant un minimum de trois jours. Les murs plombés garantissent l'entourage de l'exposition externe, les sanitaires sont reliés à des cuves de stockage pour une gestion en décroissance.

Des études ${ }^{1}$ à l'occasion de ces hospitalisations en chambres protégées et après administration de fortes activités d'iode 131 nous ont permis d'effectuer des mesures de contamination et d'exposition dues au patient.

1 Travail ayant reçu le soutien du Comité de radioprotection d'EDF. 
5.2.1 L'étude de l'élimination urinaire durant les trois jours d'hospitalisation a été effectuée sur une dizaine de patients par recueil fractionné dans le temps de la totalité des urines. Les estimations de l'activité varient relativement peu et se situent toujours entre 70 et $80 \%$ de l'activité administrée. La période effective est très variable mais cette relative imprécision est largement expliquée par le fait que les patients ont, en post-opératoire, un reliquat de thyroïde plus ou moins important selon les cas.

5.2.2 La contamination de l'atmosphère de la chambre lors de la présence du patient administré a été mesurée devant la bouche d'extraction de l'air. Les mesures ont été pratiquées sur filtre spécial à charbon actif ventilé sur $72 \mathrm{~h}$ par un système de pompe avec mise en place de deux cartouches successives pour déterminer le rendement de piégeage.

Les résultats de concentration volumique en iode 131 obtenus pour huit patients ayant séjourné $72 \mathrm{~h}$ sont portés dans le tableau suivant (Tab IV).

TABLEAU IV

Concentration volumique moyenne d'iode 131 prélevée à la bouche d'extraction Iodine 131 mean activity concentrations sampled at the air exhaust

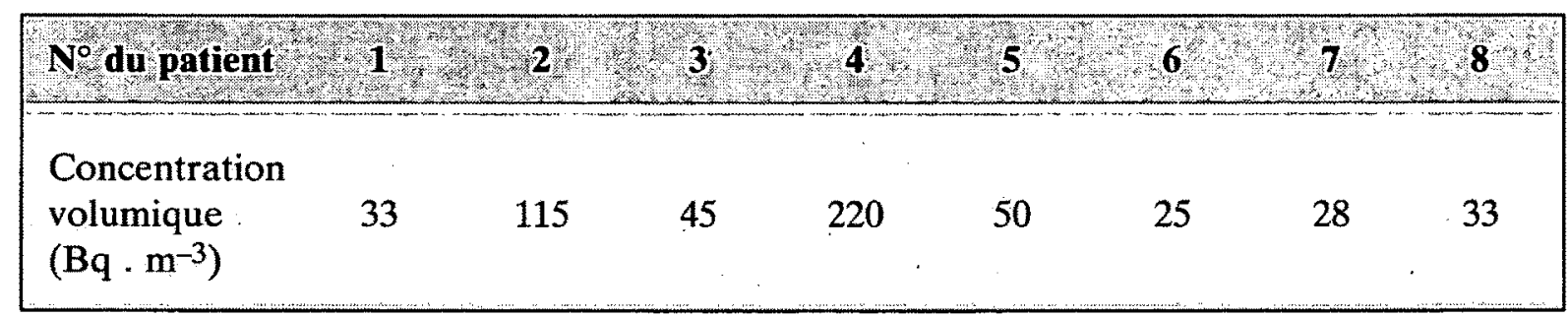

Des mesures fractionnées, rapportées à la limite dérivée de concentration de l'air (LDCA) de l'iode $131\left(700 \mathrm{~Bq} \cdot \mathrm{m}^{-3}\right)$ faites sur $24 \mathrm{~h}$ montrent que les concentrations varient :

- le 1 er jour de 0,4 à $0,8 \mathrm{LDCA}$

- le $2^{\mathrm{e}}$ jour de 0,09 à $0,13 \mathrm{LDCA}$

- le $3^{\mathrm{e}}$ jour de 0,01 à 0,04 LDCA.

Compte tenu de la perturbation du prélèvement par l'extraction de l'air de la chambre, on peut estimer que la contamination atmosphérique de la pièce est habituellement supérieure à ces valeurs.

5.2.3 Des recherches de contamination surfacique ont été pratiquées par frottis de coton hydrophile sur la surface des sols, des sanitaires et diverses poignées. Ces mesures ont été réalisées pour plus de dix patients. L'étalonnage de rendement de l'activité des frottis a été mesuré, il est de l'ordre de $50 \%$. Les comptages pour une fenêtre efficace de la sonde de $6 \mathrm{~cm}^{2}$ après étalonnage sont portés dans le tableau V. 
TABLEAU $V$

Moyenne des activités surfaciques d'iode 131 obtenue pour 10 patients Iodine 131 mean surface activities in 10 patients

\begin{tabular}{|c|c|c|c|c|}
\hline Lieu & Sol-chambre & Sol-salle de bains & $\begin{array}{c}\text { Sanitaires } \\
3\end{array}$ & $\begin{array}{c}\text { Poignées } \\
4\end{array}$ \\
\hline $\begin{array}{l}\text { Activité } \\
\text { moyenne } \\
\left(\mathrm{Bq} \cdot \mathrm{cm}^{-2}\right)\end{array}$ & 16,1 & 52,6 & 2,5 & 8,5 \\
\hline
\end{tabular}

Les frottis les plus significatifs proviennent essentiellement des taches d'urine sur le sol à proximité des toilettes. Si on tient compte du sexe des patients, on se rend compte que la contamination, notamment du sol, est plus élevée pour les hommes que pour les femmes. On peut imaginer que les projections sont plus importantes lors de la miction. En dehors des recommandations lors de la miction, les contre-mesures immédiates sont l'utilisation, par le patient, dans la chambre, de chaussures jetables et, pour le personnel, des surbottes à usage unique disposées à l'entrée de la chambre protégée. Reste posée la question de l'importance de la contamination au domicile du patient.

5.2.4 Le suivi des patients à leur sortie de chambre nous a permis d'estimer encore la source d'exposition et de contamination qu'ils constituent pour leur entourage de retour à leur domicile. Pour estimer la source nous avons mesuré l'exposition sur $24 \mathrm{~h}$ au contact de la loge thyroïdienne en disposant des pastilles de fluorure de lithium (LiF) au contact de la peau. Ces mesures ont été effectuées sur 6 patients du $4 \mathrm{e}$ au $7 \mathrm{e}$ jour après l'administration de la dose thérapeutique (Tab VI).

Compte tenu du temps et de la distance, il est possible avec ces valeurs d'obtenir l'ordre de grandeur des doses délivrées à l'entourage.

Pour juger de la contamination toujours possible nous avons proposé, après quelques jours de vie à domicile, aux membres proches du patient, conjoint, enfants, parents, de se prêter à un examen anthropogammamétrique. Les résultats obtenus sont reportés dans le tableau VII.

A l'examen des résultats, les contaminations internes sont rares, en tout cas faibles, puisque pour la plupart des personnes l'activité thyroïde est non significative. La contamination externe est plus fréquente et, sans être très importante, elle concerne essentiellement le conjoint, elle est en partie due aux vêtements. 


\section{TABLEAU VI}

Exposition externe ( $\mathrm{mSv}$ ) au contact de la thyroïde External exposure $(\mathrm{mSv})$ in contact with the thyroid

\begin{tabular}{|c|c|c|c|c|}
\hline Cas/délai & 4 jour & $5^{e}$ jour & $6^{e}$ jour & 7 e jour \\
\hline $\begin{array}{l}\text { Femme } 71 \text { ans } \\
3,7 \mathrm{GBq}\end{array}$ & $16,8 \pm 0,8$ & $13 \pm 0,2$ & $7,8 \pm 0,1$ & $1,34 \pm 0,04$ \\
\hline $\begin{array}{l}\text { Homme } 54 \text { ans } \\
1,85 \mathrm{GBq}\end{array}$ & $131 \pm 3$ & $131 \pm 0$ & $89,6 \pm 6,6$ & $83,4 \pm 2,1$ \\
\hline $\begin{array}{l}\text { Homme } 45 \text { ans } \\
5,55 \mathrm{GBq}\end{array}$ & $50,7 \pm 1,2$ & $49,4 \pm 2,05$ & $39,2 \pm 0,7$ & $15,7 \pm 0,75$ \\
\hline $\begin{array}{l}\text { Femme } 35 \text { ans } \\
3,7 \mathrm{GBq}\end{array}$ & $22,1 \pm 1,4$ & $22,8 \pm 1,7$ & $16,2 \pm 0,5$ & $11,9 \pm 0,35$ \\
\hline $\begin{array}{l}\text { Femme } 71 \text { ans } \\
3,7 \mathrm{GBq}\end{array}$ & $7,56 \pm 0,2$ & $4 \pm 0,3$ & $1,9 \pm 0,0$ & $1,8 \pm 0,3$ \\
\hline $\begin{array}{l}\text { Homme } 19 \text { ans } \\
3,7 \mathrm{GBq}\end{array}$ & $6,7 \pm 0,8$ & $3,6 \pm 0,4$ & $2,0 \pm 0,2$ & $1,5 \pm 0,0$ \\
\hline
\end{tabular}

TABLEAU VII

Résultats d'examens anthropogammamétriques réalisés chez les proches des patients. Les mesures sont données, au niveau de la thyroïde, pour des comptages corps entier, avant et après lavage Whole-body counts of patients' relatives, at the level of the thyroid, before and after washing

\begin{tabular}{|c|c|c|c|c|c|}
\hline \multirow[b]{2}{*}{ Cas } & \multirow[b]{2}{*}{ Délai } & & \multicolumn{3}{|c|}{ Activité d'iode 131 (en Bq) } \\
\hline & & & Thyroïde & $\begin{array}{l}\text { Comptage } \\
\text { avant lavage }\end{array}$ & $\begin{array}{l}\text { Comptage } \\
\text { après lavage }\end{array}$ \\
\hline $\begin{array}{l}\text { Homme } 54 \text { ans } \\
1,85 \mathrm{GBq}\end{array}$ & $11 \mathrm{j}$ & $\begin{array}{l}\text { Mère } \\
\text { Fils }\end{array}$ & $\begin{array}{l}0 \\
0\end{array}$ & $\begin{array}{l}+\varepsilon \\
+\varepsilon\end{array}$ & $\begin{array}{l}0 \\
0\end{array}$ \\
\hline $\begin{array}{l}\text { Femme } 25 \text { ans } \\
3,7 \mathrm{GBq}\end{array}$ & $13 \mathrm{j}$ & Mari & 0 & $1,036 \mathrm{kBq}$ & $0,55 \mathrm{kBq}$ \\
\hline $\begin{array}{l}\text { Homme } 45 \text { ans } \\
5,55 \mathrm{GBq}\end{array}$ & $11 \mathrm{j}$ & $\begin{array}{l}\text { Femme } \\
\text { Enfants }\end{array}$ & $\begin{array}{l}0,309 \mathrm{kBq} \\
0,148 \mathrm{kBq}\end{array}$ & 1 & 1 \\
\hline $\begin{array}{l}\text { Femme } 35 \text { ans } \\
3,7 \mathrm{GBq}\end{array}$ & $12 \mathrm{j}$ & $\begin{array}{l}\text { Mari } \\
\text { 1re fille: } \\
\text { 2e fille }\end{array}$ & $\begin{array}{c}0,148 \mathrm{kBq} \\
6 \mathrm{~Bq} \\
8 \mathrm{~Bq}\end{array}$ & $\begin{array}{c}15,84 \mathrm{kBq} \\
240 \mathrm{~Bq} \\
385 \mathrm{~Bq}\end{array}$ & $\begin{array}{c}11,32 \mathrm{kBq} \\
63 \mathrm{~Bq} \\
85 \mathrm{~Bq}\end{array}$ \\
\hline $\begin{array}{l}\text { Homme } 19 \text { ans } \\
3,7 \mathrm{GBq}\end{array}$ & $13 \mathrm{j}$ & $\begin{array}{l}\text { Mère } \\
\text { Père }\end{array}$ & $\begin{array}{c}9 \mathrm{~Bq} \\
15 \mathrm{~Bq}\end{array}$ & $\begin{array}{c}58 \mathrm{~Bq} \\
92,5 \mathrm{~Bq}\end{array}$ & $\begin{array}{l}<30 \mathrm{~Bq} \\
<30 \mathrm{~Bq}\end{array}$ \\
\hline
\end{tabular}




\section{Conclusion}

Les rejets d'effluents radioactifs en milieu hospitalier dépendent donc, du respect de la réglementation, de la bonne gestion des déchets radioactifs et des conseils de radioprotection donnés aux patients (indispensables surtout à ceux qui ont reçu de fortes activités). La désignation de la personne compétente en radioprotection marque une très probable évolution dans le respect de la réglementation par une amélioration de la mise en œuvre des moyens de radioprotection.

La bonne gestion des déchets radioactifs n'est pas, en définitive, si facile si on en juge par les $140 \mathrm{t}$ (environ) de "colis" aujourd'hui confiés à l'ANDRA par l'ensemble du secteur d'activité médicale. Les solutions de tri et de gestion préconisées par le rapport ACOMEN-ANDRA pourraient facilement réduire d'un facteur 10 ce volume (seuls les éléments de période supérieure à celle de l'iode 125 devraient être enlevés) et surtout éviteraient un tri a posteriori, onéreux et dangereux par son risque chimique et infectieux.

En définitive, après que soit assurée une bonne mise en œuvre des dispositions réglementaires, les seuls éléments "pratiques" de radioprotection pour l'environnement sont les conseils d'hygiène et de comportements que l'on doit donner aux patients pour éviter la contamination de leur entourage. Ces précautions sont importantes après administration thérapeutique, notamment lors de la miction, et particulièrement pour les hommes qui, parce qu'ils urinent debout, sont responsables d'un risque de pollution plus important.

\section{RÉFÉRENCES}

[1] ACOMEN - L'irradiation Interstitielle. Bull. ACOMEN, 1988, $\mathrm{n}^{\circ} 2$ et 3.

[2] ANDRA-ACOMEN- Gestion des déchets radioactifs produits par les hôpitaux. Lyon : Editions ACOMEN 1986.

[3] BARBIER Y., Ed. - Les Immunodosages : de la théorie à la pratique. Lyon : Editions ACOMEN, 1989

[4] FOGELMAN I., MAISEY M. - Atlas de médecine nucléaire. Paris : Medsi/McGraw-Hill, 1992.

[5] FRANCE. Protection contre les rayonnements ionisants, textes législatifs et réglementaires (Brochure $\mathrm{n}^{\circ}$ 1420). Paris : Journaux Officiels, 1994.

[6] GAMBINI D., GRANIER R. - Manuel pratique de radioprotection. Paris : Editions médicales internationales, EMI, 1992.

[7] MORETTI J.L., RIGO P., BISCHOF-DELALOYE R., TAILlEFER R., CAILlATVIGNERON N., KARCHER G. - Imagerie nucléaire fonctionnelle. Paris : Masson, 1991.

[8] NAJEAN Y., Ed. - Médecine nucléaire. Paris : Ellipses, 1990. 